\title{
CONTROL DE CONVENCIONALIDAD Y TUTELA MULTINIVEL DE DERECHOS
}

\author{
Manuel Fondevila Marón
}

doi: http://dx.doi.org/10.18543/ed-65(1)-2017pp347-360

\begin{abstract}
SUMARIO: 1. INTRODUCCIÓN METODOLÓGICA. 2. EN CONTROL DIFUSO DE CONVENCIONALIDAD EN EL SISTEMA AMERICANO DE PROTECCIÓN DE DERECHOS Humanos Y LA ALTERNATIVA EUROPEA. 3. LOS PROTOCOLOS $15 \mathrm{Y}$ 16 DE REFORMA DEL CEDH: HACÍA UN MODELO CONCENTRADO DE CONVENCIONALIDAD. 4. LA PROBLEMÁTICA EN EL CASO ESPAÑOL. 5. PROPUESTA DE PROTECCIÓN MULTINIVEL DE DERECHOS. 6. CONCLUSIONES.
\end{abstract}

\section{INTRODUCCIÓN METODOLÓGICA}

El constitucionalismo moderno es una realidad compleja ${ }^{1}$, que exige por parte de todos los operadores jurídicos un esfuerzo para superar los retos que se plantean, a fin de que siga respetándose su verdadera esencia, esto es, el objetivo de alcanzar cada vez mayores cuotas de Democracia y Libertad $^{2}$.

Sin duda alguna, uno de esos retos no es sino la existencia, a nivel local, estatal, e internacional, de catálogos de derechos a los que, en virtud del principio de progresividad debe dotárseles de eficacia y realidad. Para algunos autores esta positivización excesiva supone ya una merma de la fuerza

${ }^{1}$ Cfr., por todos, Jose Joaquim Gomes Canotilho, Direito Constitucional e Teoria da Constituição, (Coimbra: Almeida, 2003), 1335.

2 En este sentido Pedro De Vega García, «Mundialización y Derecho Constitucional: la crisis del principio democrático en el constitucionalismo actual». Revista de Estudios Políticos, n. ${ }^{\circ} 100$ (1998): 33. 
implícita en la noción de derecho ${ }^{3}$ que, evidentemente, será mayor si no se les dota de adecuadas garantías, condenándolos al ámbito de la retórica.

En este trabajo vamos a ocuparnos tan sólo de un aspecto no menor de esta problemática como es el control de convencionalidad, a nivel nacional, de los derechos reconocidos en los Tratados Internacionales de Derechos Humanos. Desde el punto de vista teórico y práctico consideramos imprescindible, para el desarrollo de este trabajo, la utilización de:

a) La metodología propia del Derecho Constitucional Comparado: la adopción de una perspectiva comparada servirá no sólo para buscar soluciones a los problemas jurídicos en el Derecho extranjero, sino -y más importante- para un replanteamiento de modelos existentes, fundamentalmente, de justicia constitucional.

Para los fines y propósitos de este trabajo afirmar que el jurista de hoy tiene necesariamente que ser comparatista ${ }^{4}$ implica ser conscientes, además, de que las categorías jurídicas de los sistemas jurídicos comparados han evolucionado y cambiado; y ello es importante, como veremos, porque la mayor dificultad que parece existir en Europa para que los jueces ordinarios hagan un control de convencionalidad difuso es la aparente contradicción -que en nuestra opinión no es tal-con el control de constitucionalidad concentrado.

El error consiste en aferrarse a viejas categorías, como la de «modelo concentrado de control de constitucionalidad» (por oposición al control difuso norteamericano) que deberían -con una perspectiva de Derecho Comparado- repensarse, teniendo en cuenta que la mayoría de los modelos de justicia constitucional actualmente pueden se consideran $«$ mixtos $»^{5}$.

La dicotomía modelo concentrado/modelo difuso, sobre todo cuando comparamos continentes, tiene, como señala el Profesor L. Pegoraro, tantas excepciones que no resulta operativa. En Europa, por ejemplo, domina el modelo de control de constitucionalidad concentrado de origen austríaco, pero además de que en algunos países, como España, han incluido elementos de cierto control difuso (la cuestión de constitucionalidad) en Portugal existe un verdadero control difuso y un control concentrado en manos del Tribunal Constitucional. En Francia, hasta 2008, el control de constitucionalidad era de carácter político.

${ }^{3}$ Cfr. Santiago SÁnchez GonzÁlez, «¿Todavía más derechos? ¿De qué derechos hablamos?», Teoría y Realidad Constitucional, n. ${ }^{\circ} 25$ (2010): 304.

${ }^{4}$ Cfr. Prefazione de Lucio Pegoraro y Angelo Rinella, Diritto pubblico comparato. Profili metodologici (Padova: CEDAM, 2007).

5 Cfr. Lucio Pegoraro, La justicia constitucional. Una perspectiva comparada, (Madrid: Dykinson, 2004), 167. 
En cuanto a América Latina, algunos países como Brasil o el Salvador también tienen un control de constitucional difuso y otro concentrado en manos del Tribunal Supremo. En Ecuador el control de constitucionalidad, por la interpretación que del artículo 428 de su Constitución ha hecho la Corte Constitucional, existe control concentrado. En Chile y en Paraguay, está dividido entre el control inter partes de la Corte Suprema y el control erga homnes el Tribunal Constitucional o Sala Constitucional de aquel Tribunal respectivamente.

Por lo tanto, no puede ser la relación con el sistema de control de constitucionalidad el que haga perder, a través de una no realización del de convencionalidad, la validez en la práctica de los Tratados de Derechos Humanos.

b) Una perspectiva supranacional, propia del llamado Derecho Constitucional Internacional, conciliadora del Derecho Constitucional y el Derecho Internacional.

Por Derecho Constitucional Internacional entendemos el fenómeno por el que las Constituciones de los Estados Democráticos constituyen la mejor garantía del Derecho Internacional y, en concreto, del Derecho Internacional humanitario. Así pues, como indica MirkineGuetzèvitch, «la fuerza obligatoria de los Tratados Internacionales está en directa dependencia de la forma de gobierno de los Estados contratantes ${ }^{6}$, precisamente porque éstas se internacionalizan a la vez que los Tratados Internacionales son receptores a su vez de las tradiciones constitucionales comunes. De este modo habla nuestro autor de unidad y no de primacía del Derecho Constitucional sobre el Internacional o viceversa ${ }^{7}$. Además, a diferencia de lo que sostienen erróneamente algunos tratadistas de Derecho Internacional, de este modo no se pone en cuestión la soberanía de los Estados ${ }^{8}$.

Como en nuestro trabajo vamos a relacionar la Justicia Constitucional con los Tratados Internacionales, nos interesa destacar que a pesar de que la problemática del control de convencionalidad de los Tratados Internacionales es reciente ${ }^{9}$, comenzando - como vamos a ver a continuación- a finales de la década de los años 90, una primera teorización la encontramos en este autor de los años 30, cuando afirma: «El

${ }^{6}$ Boris Mirkine-Guetzèvitch, Derecho Constitucional Internacional, (Madrid: Reus, 2008), 120.

7 Mirkine-GuezÈvitch, Derecho Constitucional Internacional, 471.

${ }^{8}$ Cfr. Favia Piovesan, Direitos Humanos e o Direito Constitucional Internacional, (São Paulo:Saraiba), 83-89.

9 Cfr. Valerio Mazzuoli, O controle jurisdicional da convencionalidade das leis. 2 (São Paulo: Revista dos Tribunais, 2011), 73. 
problema de la validez interna de los Tratados Internacionales recibe una solución constitucional particular en los Estados en los que funciona la justicia constitucional [ahora prácticamente todos]. Si la Constitución reconoce al Derecho Constitucional como parte integrante del Derecho nacional, y si en este funciona la justicia constitucional, el conflicto entre la Ley y el Tratado no puede tener más que una solución: la primacía del Tratado» ${ }^{10}$.

Una de las mayores dificultades, sin embargo, que nos encontramos en el Derecho Comparado no es otra que la posición de los Tratados de Derechos Humanos no es igual en todos lugares, pudiendo distinguir, al menos: 1) Algún Estado, como en Holanda, donde los Tratados tienen una autoridad superior a la Constitución ${ }^{11}$; 2) En otros Estados, como es el caso de Austria ${ }^{12}$ o Argentina ${ }^{13}$, los Tratados conforman el bloque de constitucionalidad, teniendo la misma fuerza que la propia Constitución; 3) En otros Estados, como es el caso de Francia ${ }^{14}$ o México ${ }^{15}$, los Tratados se sitúan por encima de las leyes; 4) En Estados como Alemania ${ }^{16}$ los Tratados equivalen a una ley ordinaria.

La tendencia mayoritaria en la doctrina, sin embargo, y la hipótesis de nuestro trabajo, es que éstos deban tener un valor supralegal.

\section{EN CONTROL DIFUSO DE CONVENCIONALIDAD EN EL SISTEMA AMERICANO DE PROTECCIÓN DE DERECHOS HUMANOS Y LA ALTERNATIVA EUROPEA.}

El llamado control difuso de convencionalidad es una creación jurídica de la Corte Interamericana, la cual, basándose en el art. 2 del Pacto de San José, impone a los jueces nacionales la obligación de confrontar su legislación

${ }^{10}$ Mirkine-Gueż̀VITCH, Derecho Constitucional Internacional, 298.

${ }^{11}$ El art. 91.3 establece: «Cuando un tratado contuviere estipulaciones que deroguen la Constitución o que impongan la necesidad de tal derogación, se requerirá para su adopción por las Cámaras una mayoría de al menos dos tercios del número de votos emitido». Se puede pensar que más que situar a los Tratados por encima de las leyes se trata de reformas constitucionales, pues es la misma mayoría que se exige para la modificación del Texto Fundamental. Pero considero que lo clave es la aprobación del Tratado, con esa mayoría, la que deroga la Constitución sin que, por supuesto, quepa después control a posteriori.

${ }_{12}$ El art. 50.3 obliga a considerar este tipo de Tratados como enmiendas constitucionales.

13 Art. 75.22.

14 Art. 55.

15 Así lo ha reconocido la Suprema Corte en la Tesis IX/2007 (Aislada) del Pleno. Semanario Judicial de la Federación y su Gaceta. XXV abril 2007. P. 6. Antes de este año el art. 133 se interpretaba en el sentido en que los Tratados tenían un valor incluso inferior a las leyes.

${ }_{16}$ Art. 59. 
nacional con las disposiciones de la CIDH. Establece, en concreto, bajo el título de «deber de adoptar decisiones de Derecho interno» que: «si el ejercicio de los derechos y libertades mencionados en el artículo 1 no estuviere ya garantizado por disposiciones legislativas o de otro carácter, los Estados Partes se comprometen a adoptar, con arreglo a sus procedimientos constitucionales y a las disposiciones de esta Convención, las medidas legislativas o de otro carácter que fueren necesarias para hacer efectivos tales derechos y libertades».

Esta obligación surge, en concreto, con la bien conocida Sentencia Almonacid Arellano y otros vs. Gobierno de Chile, de 2006, donde indica que «cuando un Estado ha ratificado un Tratado internacional como la Convención Americana, sus jueces, como parte del aparato del Estado, también están sometidos a ella, lo que les obliga a velar porque los efectos de las disposiciones de la Convención no se vean mermadas por la aplicación de leyes contrarias a su objeto y fin, y que desde un inicio carecen de efectos jurídicos», aunque matizaba en Trabajadores cesados del Congreso vs. Perú, de 24 del mismo año, que ello «tampoco implica que ese control deba ejercerse siempre, sin considerar otros presupuestos formales y materiales de admisibilidad y procedencia de ese tipo de acciones».

Aunque no es menester, en un trabajo de estas características, un estudio exhaustivo de los casos, conviene indicar que estas dos sentencias vienen precedidas de algunos antecedentes que fueron, poco a poco, construyendo la idea de que las leyes internas deben ser sometidas a un control respecto de la Convención.

En los casos Loayza Tamayo versus Perú (1997) o Suárez Rosero versus Ecuador (1997) lo característico es que la Corte considera ciertas normativas internas como contrarias a la Convención, pero no expresa claramente que deban ser anuladas. En los casos de «La última tentación de Cristo» (Olmedo y Bustos contra Chile (2001)) y Barrios Altos versus Perú (2001) la Corte Interamericana reconoce ya que las normas internas que evitan la aplicación de la Convención no pueden seguir un obstáculo a la eficacia del Tratado.

Por su parte, la expresión «control de convencionalidad», aparece por primera vez en el voto concurrente del Juez García Ramírez en el caso Myrna Mack Chang versus Guatemala (2003).

De este modo podemos separar dos etapas en las que, hasta 2006, existía un control de convencionalidad (aunque no se le llamase así) que algunos autores han denominado desde arriba, mientras que, desde 2006, convierte la Corte a los jueces nacionales en colaboradores suyos ${ }^{17}$. Por esta razón hablamos, en el caso del continente americano, de control difuso de convencionalidad: todos

${ }^{17}$ Cfr. Nestor Pedro SAgÜEs, «El «control de convencionalidad» en el sistema interamericano, y sus anticipos en el ámbito de los derechos económicos-sociales. Concordancias y diferencias con el sistema europeo». En Construcción y Papel de los Derechos 
los jueces están facultados para realizar este examen de convencionalidad de las leyes e inaplicarlas en caso de que resulte negativo.

Nada de esto ha ocurrido, por el contrario, en el sistema europeo de Derechos Humanos. Lo cierto es que la vaguedad del art. 1 del CEDH, el cual establece, simplemente, que «las Altas Partes Contratantes reconocen a toda persona bajo su jurisdicción los derechos y libertades definidos en el Título I del presente Convenio» supone, efectivamente, un mal punto de partida para que el Tribunal europeo pueda llegar a una conclusión similar ${ }^{18}$.

Sin embargo, ello no es óbice para que, de acuerdo con instrumentos de legislación interna, exista la posibilidad de un control difuso de convencionalidad en el continente europeo. De hecho, a falta, hasta 2008, de un verdadero control de constitucionalidad de las leyes, este control de convencionalidad ya se aplica normalmente en Francia con base en lo dispuesto en el artículo 55 de su Constitución. En otros países, como España, no está generalizado, pero sin embargo algunos jueces y Tribunales lo han ejercido de manera puntual ${ }^{19}$.

Es decir, que el control (difuso) de convencionalidad es una alternativa por la que la legislación y la jurisprudencia nacional pueden optar dentro de la comprensión unitaria del Derecho Público (Constitucional e Internacional) y para dotar de mayor garantía a los derechos. Está claro que donde los Tratados Internacionales tengan valor legal las dificultades interpretativas son mayores; no así donde se entienda que tienen un valor supralegal.

Con todo, el sistema de protección de Derechos Humanos europeo va a establecer, además, un control concentrado de convencionalidad.

\section{LOS PROTOCOLOS 15 Y 16 DE REFORMA DEL CEDH: HACÍA UN MODELO CONCENTRADO DE CONVENCIONALIDAD}

En el sistema europeo de protección de Derechos Humanos se trabaja, en dos líneas diversas, establecidas respectivamente en los protocolos $n .{ }^{\circ} 15 \mathrm{y}$

Sociales Fundamentales, ed. por Armin Bogdandy (México: Universidad Nacional Autónoma de México, Instituto de Investigaciones Jurídicas, 2011), 381-382.

${ }^{18}$ Cfr., Raul Canosa UsERa, El control de convencionalidad (Navarra, Thompsom Reuters, 2015), 58.

19 Por ejemplo, cita, en este sentido el Profesor R. Canosa cinco casos bastante ejemplificativos: 1) STS de 8 de Julio de 2014, haciendo una «interpretación conforme» a los Tratados del art. 89 de la L.O 11/2003. Este caso no es propiamente un control de convencionalidad, pues no deja de aplicarla la normativa interna como si hacen 2) la Sentencia del Juzgado de lo Social n. ${ }^{\circ} 2$ de Barcelona, de 19 de noviembre de 2013; 3) Sentencia del Juzgado de lo Social n. ${ }^{\circ} 1$ de Tarragona, de 2 de abril de 2014; 4) Sentencia del Juzgado de lo Social n. ${ }^{\circ} 1$ de Mataró, de 29 de abril del 2014; 5) Sentencia del Juzgado de lo Social n. ${ }^{\circ} 1$ de Toledo de 27 de noviembre de 2014. En todos estos casos, por considerar la normativa interna contraria a la CSE (Cfr., CANOSA, El control de convencionalidad, 101 y ss.). 
16 de reforma del Convenio Europeo de Derechos Humanos, distintas a las adoptadas por la CIDH.

a) El protocolo n. ${ }^{\circ} 15$ establece, en su artículo 1, que se incluirá en el preámbulo el principio de subsidiariedad y el margen de apreciación nacional de los Estados. Además (art. 4), se reduce el plazo para la admisibilidad del recurso de seis a cuatro meses a la vez que se objetiviza el recurso, en tanto que (art. 5) que serán inadmitidas aquellas demandas en las que el actor no haya sufrido un perjuicio importante.

b) El protocolo n. ${ }^{\circ} 16$ parece caminar hacía un control concentrado de convencionalidad por cuanto establece que los Altos Tribunales de los Estados Miembros podrán presentarle opiniones consultivas sobre la interpretación de las disposiciones del Tratado.

Hablamos de control concentrado de convencionalidad, porque a) por un lado, que aumenta la responsabilidad de los Estados en la protección de los derechos (ya que el protocolo . $^{\circ} 15$ establece requisitos objetivos más rígidos para el acceso al Tribunal); b) de cualquier modo, sigue vigente, si esta protección fallase dentro de los Estados, el acceso directo a los ciudadanos que estableció el protocolo $\mathrm{n}^{\circ}{ }^{\mathrm{o}} 11$; c) aunque esta posibilidad de opiniones consultivas existe ya en el ámbito de la $\mathrm{CIDH}^{20}, \mathrm{y}$ ni allí ni aquí tienen efecto vinculante $^{21}$, existe la importante diferencia de que allí son los Estados parte, o los órganos mencionados en el Capítulo X de la Carta de la OEA, y no los «Altos Tribunales» de los Estados Miembros los que pueden pedir dicha opinión consultiva. Por esta razón, aunque la falta de efecto vinculante lleva a la doctrina americana a distinguir entre estas opiniones consultivas y el control de convencionalidad ${ }^{22}$, en Europa se puede decir que la situación es distinta.

En concreto, el art. 1 del protocolo $n .^{\circ} 16$ establece: «1. Highest courts and tribunals of a High Contracting Party, as specified in accordance with Article 10, may request the Court to give advisory opinions on questions of principle relating to the interpretation or application of the rights and freedoms defined in the Convention or the protocols thereto (...) 2 . The requesting court or tribunal may seek an advisory opinion only in the context of a case pending before it. (...) 3. The requesting court or tribunal shall give reasons for its request and shall provide the relevant legal and factual background of the pending case».

En un sistema de control concentrado de constitucionalidad, la primera y más básica dificultad que existe para aplicar el control de convencionalidad

${ }^{20}$ Vid., art. 64 CIDH.

${ }^{21}$ Vid., art. 5 protocolo 16.

22 Vid., Mazzuoli O controle jurisdiccional..., 92. 
es que, en principio, los jueces están vinculados a la ley, y no pueden dejar de aplicarla por interpretar que son contrarias a la Constitución o a los Tratados Internacionales.

A esta primera, básica y medular dificultad se suman otras dificultades conexas, también de gran importancia:

a) En primer lugar, la naturaleza y posición que ocupan los Tratados Internacionales y, en concreto, los relativos a Derechos Fundamentales, a los que ya hemos hecho referencia en la introducción.

b) En segundo lugar, la jurisprudencia de los Tribunales Constitucionales y su mayor o menor disposición a hacer una recepción de la jurisprudencia del TEDH en materia de Derechos Humanos.

En el próximo epígrafe, tomando como ejemplo el caso de España, veremos cómo pueden resolverse esas dificultades y como no hacerlo genera otras.

\section{LA PROBLEMÁTICA EN EL CASO ESPAÑOL}

Por cuanto respecta a la primera cuestión, aunque el art. 96 CE no establece la posición jerárquica de los Tratados en España, doctrinal y legislativamente (Ley 25/2014), tal y como también lo entendía el Consejo de Estado $^{23}$, hay acuerdo generalizado en que tienen valor supralegal. De este modo la dificultad podemos decir que es prácticamente inexistente.

Mayor dificultad presenta la segunda cuestión. El TC, aunque emplea a menudo la jurisprudencia del TEDH en sus fundamentos jurídicos, tiene una consolidada y constante jurisprudencia en el sentido de no entender el CEDH como parámetro de enjuiciamiento de constitucionalidad de las leyes. El supremo custodio constitucional hace una recepción del Convenio de Roma y la jurisprudencia del TEDH para interpretar e identificar el contenido de los Derechos Fundamentales, pero no constituyen un canon de validez de las normas y actos de los poderes públicos, argumentando, además, que si así fuese, sobraría la declaración constitucional ${ }^{24}$. Algunas veces, la labor interpretativa que realiza el Tribunal Constitucional es muy generosa, a fin de evitar una posible condena del Estado por el TEDH, pero son precisamente estos casos que se aproximan a un verdadero control de convencionalidad los que confirman la regla ${ }^{25}$.

${ }^{23}$ En su Memoria anual de 2007 (p. 149), especifica que venía junto al tradicional control de constitucionalidad de los proyectos legislativos, realiza un «control de convencionalidad».

${ }^{24}$ SSTC 64/1991 y 236/2007, entre otras.

${ }^{25}$ Por ejemplo, en la STC 11/2016 dotó de un nuevo contenido al art. 18.1 CE (referido a la «intimidad privada y familiar», incluyendo dentro de éste, y basándose en la ju- 
Señalar las dificultades para llevar a cabo el control de convencionalidad no puede desembocar en dejar de realizarlo, pues aún si el TEDH no lo impone a los Estados firmantes, resulta una exigencia derivada del principio del Derecho Internacional pacta sunt servanda y de la propia Constitución.

De esto parece ser consciente la mejor doctrina, y recientemente se han propuesto dos tipos de soluciones:

a) El Profesor L. Jimena ${ }^{26}$, con quien coincidimos plenamente propone que este control de convencionalidad sea realizado por los jueces ordinarios, como en el sistema americano de protección de Derechos Humanos. Se basa en lo dispuesto en los artículos 9.3, 10.2, 96 de la Constitución y en el art. 5 y 6 LOPJ.

b) El Profesor Raúl Canosa, sin embargo, plantea en la obra citada que resulta inconveniente un control de convencionalidad difuso, y propone una reforma constitucional para que sea el supremo intérprete constitucional quien lleve a cabo el control concentrado de convencionalidad lo que, a su juicio, es la dirección tomada por el sistema europeo de protección de Derechos Humanos.

Ni que decir tiene que considerar únicamente al Tribunal Constitucional hight court no va ayudar en las relaciones entre los dos Tribunales, que no siempre fueron buenas, y que parece que comenzaban a encaminarse en los últimos años.

La primera postura parece mostrar menos dificultades y, de hecho, es perfectamente posible ponerla en práctica del mismo modo que cuando todos los jueces nacionales, actuando como jueces de la UE, inaplican una ley interna en virtud del principio de primacía.

La segunda conlleva, en primer lugar, una reforma de la Constitución, que es algo muy difícil de llevar a la práctica en España ${ }^{27} \mathrm{y}$, en segundo lugar, una sobrecarga de trabajo para el TC y el propio TEDH que, precisamente, la L.O 6/2007 y el protocolo n. $^{\circ} 15$ respectivamente tratan de evitar.

risprudencia del TEDH sobre el más amplio «derecho a la vida privada familiar» art. 8 $\mathrm{CEDH}$, el derecho de los padres a despedirse de los restos mortales de una criatura abortiva. La solución adoptada fue criticada por los votos particulares de los Magistrados Francisco de los Cobos y Encarnación Roca Trías.

${ }^{26}$ Cfr., Luis Jimena QueSADA, Jurisdicción nacional y control de convencionalidad. A propósito del diálogo judicial global y de la tutela multinivel de derechos, (Navarra: Thompsom Reuters, 2013) passim.

27 Sobre esto Cfr., Javier RUIPÉREZ AlAmillo, Reforma versus Revolución. Consideraciones desde la Teoría del Estado y de la Constitución sobre los límites materiales a la revisión constitucional, (México: Porrúa, 2014), 77 a 143 y Manuel Fondevila Marón, La reforma constitucional en España. Un ensayo de Derecho Constitucional como Ciencia Teórico-Práctica. (Santiago de Compostela: Andavira. 2016), 97 a 102. 
En una materia, además, como los Derechos Humanos, en las que están en juego las necesidades más básicas de las personas parece, como mínimo, una falta de sensibilidad obligar al juez a quo a plantear la cuestión al TC que, si se ratificase el protocolo n. $^{\circ} 16$, podría derivarla al TEDH demorando así en varios años su resolución. Si el juez decidiese no evacuar la cuestión al TC, el actor se vería obligado a plantear recursos que podrían acabar, precisamente, en el TC (cuyo acceso en amparo se ha limitado sobremanera en la L.O 6/2007) obligándole, también después de varios años, a plantear su caso ante el TEDH que empieza ya -esto es lo que intenta evitar el protocolo 15-a estar sobresaturado.

No hace falta, por tanto, un gran esfuerzo interpretativo y de voluntad político-jurídica, sobre la base de preceptos legales ya existentes, para comprender la protección multinivel de derechos desde el ámbito regional al supranacional, entendido este último tanto en el ámbito estricto de la Unión Europea como en el del Consejo de Europa. Es lo que proponemos a continuación.

\section{PROPUESTA DE PROTECCIÓN MULTINIVEL DE DERECHOS}

La existencia de declaraciones de derechos a nivel regional, estatal y supranacional supone, como ya dijimos, un reto que supone un esfuerzo para integrarlas dentro del sistema de fuentes con plena validez jurídica.

Nuestra tesis es que todo juez (estatal, nacional, comunitario ${ }^{28}$ ), debe realizar, en un juicio de Derechos Humanos o Fundamentales dentro de sus competencias, en primer lugar, un control de convencionalidad, pues si la norma o acto lesiona los derechos recogidos en un Tratado debe ser inaplicado, tanto en virtud del principio pacta sunt servanda como, tal y como venimos defendiendo, por mandato constitucional. Aunque la ley pudiese ser constitucional (y además debe presumirse que lo es), sería inaplicable -insistimos, por voluntad constitucional- por el mero hecho de ser contraria a un Tratado.

Si la norma o el acto del poder público son conformes a los Tratados Internacionales en materia de Derechos Humanos, aun le resta al juez llevar a cabo el control de constitucionalidad y, en su caso (si estamos ante un supuesto de aplicación del Derecho de la Unión Europea), el control de la

${ }^{28}$ Las reglas que van a exponerse a continuación sirven, en principio, también para el juez de la Unión Europea, una vez que la UE se adhiera al CEDH. Deberá hacer, primero, el control de convencionalidad de la normativa europea y, después, confrontarla con los derechos reconocidos en la Carta de Niza (elevando cuestión prejudicial). Resulta, cuanto menos, criticable, la postura proteccionista de su propia competencia mantenida por el TJUE en el Dictamen 2/13 de 18 de junio de 2014 considerando el Acuerdo de adhesión contrario a los Tratados. 
normativa respecto de los derechos reconocidos en la Carta de Niza de 2000. En este supuesto, el control que hace el juez comunitario del respeto de la normativa de los derechos contenidos en la Carta de Derechos Fundamentales de la Unión Europea sustituye, en condiciones de normalidad ${ }^{29}$, al posible amparo de los Derechos Fundamentales recogidos en la Constitución ${ }^{30}$.

${ }^{29}$ En el caso Bananas de 7 de Junio de 2000, el Tribunal Constitucional Federal alemán afirma que los recursos de amparo y las cuestiones de inconstitucionalidad de Tribunales que aleguen una lesión de los Derechos Fundamentales de la Constitución por parte del Derecho de la Unión Europea no son admisibles ab initio cuando su fundamentación no evidencia que la evolución jurídica europea, incluyendo la jurisprudencia del Tribunal de Justicia de las Comunidades Europeas tras la publicación de la Sentencia Solange II no haya alcanzado el estándar necesario de protección de los Derechos Fundamentales. Por ese motivo, el amparo y la cuestión deben evidenciar que la protección de los derechos que se considera indispensable no se garantiza con carácter general, lo que exige una contraposición de ambos ordenamientos jurídicos. Sólo en estos casos excepcionales cabría un recurso de amparo o una cuestión de inconstitucionalidad de normativa que aplica el Derecho de la Unión.

También nuestro TC, en la STC 58/2004 admite esa posibilidad excepcional.

${ }^{30}$ En algunos países, como Francia, se establece un control incidental de constitucionalidad que tiene carácter preferente. A este respecto el TJUE ha establecido, de un modo muy respetuoso con la legislación procesal de los Estados, que: que aunque el artículo 267 TFUE se opone a una normativa de un Estado miembro que establece un procedimiento incidental de control de constitucionalidad de las leyes nacionales, en la medida en que el carácter prioritario de ese procedimiento tenga como efecto impedir, tanto antes de la remisión de una cuestión de constitucionalidad al órgano jurisdiccional nacional competente para ejercer el control de constitucionalidad de las leyes como, en su caso, después de la resolución del citado órgano sobre dicha cuestión, que todos los demás órganos jurisdiccionales nacionales ejerzan su facultad o cumplan su obligación de plantear cuestiones prejudiciales al Tribunal de Justicia; este artículo no se opone, en cambio, a dicha legislación nacional, siempre que los demás órganos jurisdiccionales nacionales sigan estando facultados para: a) plantear al Tribunal de Justicia toda cuestión prejudicial que consideren necesaria, en cualquier momento del procedimiento que estimen apropiado, e incluso una vez finalizado el procedimiento incidental de control de constitucionalidad; b) adoptar toda medida necesaria para asegurar la tutela judicial provisional de los derechos conferidos por el ordenamiento jurídico de la Unión; c) dejar inaplicada, una vez finalizado ese procedimiento incidental, la disposición legislativa nacional controvertida si la consideran contraria al Derecho de la Unión; y añade - por último- que incumbe al tribunal remitente verificar si la legislación nacional controvertida en los asuntos principales puede interpretarse conforme a esas exigencias del Derecho de la Unión (Vid., STJUE Aziz Melki (asunto C-188/10)).

En nuestra humilde opinión, el control incidental de constitucionalidad, tanto antes como después de haber planteado la cuestión prejudicial puede dar lugar -en caso de eventual declaración de inconstitucionalidad de un acto que el TJUE no haya considerado contrario a los Tratados- a un conflicto que desde la perspectiva de conciliación del Derecho Constitucional y el Derecho de la Unión Europea que defendemos no debería producirse. 
No encontrándonos ante un supuesto de aplicación del Derecho Comunitario, si el juez lo es de un Estado federal en cuyas constituciones estatales se reconocen derechos y se establece normas procesal-constitucionales para su protección, deberá confrontar la norma o acto del poder público con respecto a éstas, considerando que la regla general es que la constitución estatal puede ampliar, nunca reducir, los derechos reconocidos a nivel federal.

Dependerá de cómo se configure el ordenamiento interno que pueda, por su propia autoridad, declarar la inconstitucionalidad de la norma o deba plantear la cuestión de inconstitucionalidad.

Desde la comprensión internacional del Derecho Constitucional y la perspectiva comparada que defendemos, nuestra propuesta no debería suponer ningún problema cuando de lo que se trata es de anular una ley o acto normativo por ser contraria a los Tratados, o, en su caso, a la Constitución federal o estatal. Recuérdese, a este respecto, que tanto el Convenio Europeo de Derechos Humanos como el Pacto de San José establecen que ninguna disposición contenida en ellos se entenderá restrictiva de los Derechos Fundamentales reconocidos a nivel nacional ${ }^{31}$. Tampoco plantearán ningún problema los juicios de Derechos Humanos entre particulares, pues los derechos recogidos en la Constitución deberán interpretarse conforme a los Tratados.

Todo ello sin perjuicio de un posible control de constitucionalidad $a$ posteriori de los Tratados (donde la Constitución lo permita como es el caso, por ejemplo, de España ${ }^{32}$ y Alemania ${ }^{33}$ ). Es posible que el custodio constitucional entienda que un derecho reconocido en un Tratado es contrario a la Constitución. Cuando el conflicto entre la Constitución y el Tratado sea insalvable por vía de la interpretación, el principio democrático en el que se sustenta la supremacía constitucional debe hacer prevalecer a aquélla, pues si los Tratados han sido aprobados en virtud de facultades derivadas de la propia Constitución, no cabe defender que aquellos puedan servir para destruirla. Esta última posibilidad debe ser absolutamente excepcional: una auténtica hipótesis de laboratorio que, además, en coherencia, debería implicar la salida del Estado organismo supranacional. Aunque es esperable que no ocurra jamás, es una posibilidad que, de acuerdo con el principio de supremacía constitucional, está siempre abierta al Poder Constituyente ${ }^{34}$.

31 Art. 53 de la Carta de Roma y art. 29 del Pacto de San José.

32 DTC $1 / 2004$.

33 Resolución de 7 de junio de 2000 (caso Bananas).

${ }^{34}$ No es cierto, por tanto, que sea imposible dar marcha atrás en el proceso de integración, tal y como recientemente ha defendido Muñoz Machado en Vieja y Nueva Constitución, Madrid: Crítica, 2015, Epígrafe X. En contra de sus tesis, remito a Manuel FondE- 


\section{CONCLUSIONES}

En este ensayo hemos sostenido, básicamente, que la mejor protección de los derechos se da desde una protección multinivel que incluya desde los derechos reconocidos en las constituciones o estatutos regionales, a aquellos reconocidos a nivel supranacional.

Los dos primeros dependen, obviamente, tan sólo de los mecanismos de control de constitucionalidad a nivel nacional y regional recogidos a nivel interno.

A nivel supranacional pueden ocurrir dos cosas: a) que bien por imposición de los Tratados, o de la interpretación que de ellos se haga por los Tribunales Supranacionales (casos Costa de 1964 del TJUE y Almonacid de 2006 de la CIDH) o bien de la normativa constitucional, y de la interpretación que de ésta hagan los jueces nacionales sobre la posición jerárquica de los Tratados, que aquí hemos defendido por encima de la ley y por debajo de la constitución, los jueces estén obligados a realizar el control de convencionalidad o; b) que la Constitución y las leyes procesales internas, así como los Tratados Internacionales guarden silencio al respecto.

En el caso concreto del Consejo de Europa, dado que el TEDH no ha impuesto a los jueces nacionales la realización del control difuso de convencionalidad, el protocolo n. ${ }^{\circ} 16$ puede servir para establecer un control de convencionalidad allí donde las normas internas no permiten de otro tipo.

\section{TITLE: Conventionality Control and multilevel tutoring of rights}

RESUMEN: En el presente trabajo hacemos un estudio comparativo del control difuso de convencionalidad en América Latina y el incipiente control concentrado de convencionalidad que, con la aprobación de los protocolos 15 y 16, está intentando establecerse en el ámbito del sistema de protección de Derechos Humanos europeo. Sostenemos, en concreto, la tesis de que el control difuso es posible en los países europeos, y en concreto en España, y que el juez ordinario debe realizar un triple o cuádruple estudio de la ley antes de su aplicación.

Palabras CLAVE: Derecho Constitucional Internacional, Posición de los Tratados, Protocolos n. ${ }^{\circ} 15$ y 16.

ABSTRACT: In the present paper, we make a comparative study of the diffuse control of conventionality in Latin America and the incipient concentrated control of conventionality, which, with the approval of protocols 15 and 16, is trying to establish itself within the scope of the European Human

VILA MARÓN, La disolución de la soberanía en el ámbito estatal. El proceso de integración europea. (Madrid: Reus, 2014), 436 y ss. 
Rights protection system. We hold, in particular, the thesis that diffuse control is possible in European countries, and specifically in Spain, and that the ordinary judge must carry out a triple or quadruple study of the law before its application.

KEY WORDS: Constitutional International Law, Treaties position, Protocols $n .^{\circ} 15$ and 16.

RECIBIDO: 28.03.2017

ACEPTADO: 30.05 .2017 